\title{
Design Optimisation of Separate-Jet Exhausts for the Next Generation of Civil Aero-Engines
}

\author{
loannis Goulos * \\ John Otter, Tomasz Stankowski and David MacManus \\ Cranfield University \\ Propulsion Engineering Centre \\ Cranfield, Bedford, MK43 OAL \\ United Kingdom \\ Nicholas Grech and Christopher Sheaf \\ Installation Aerodynamics \\ Rolls-Royce plc \\ Derby, DE24 8BJ \\ United Kingdom
}

\section{ABSTRACT}

This paper presents the development and application of a computational framework for the aerodynamic design of separate-jet exhaust systems for Very-High-Bypass-Ratio (VHBR) gas-turbine aero-engines. An analytical approach is synthesised comprising a series of fundamental modelling methods. These address the aspects of engine performance simulation, parametric geometry definition, viscous/compressible flow solution, design space exploration, and genetic optimisation. Parametric design is carried out based on minimal user-input combined with the cycle data established using a zero-dimensional (0D) engine analysis method. A mathematical approach is developed based on Class-Shape Transformation (CST) functions for the parametric geometry definition of gas-turbine exhaust components such as annular ducts, nozzles, after-bodies, and plugs. This proposed geometry formulation is coupled with an automated mesh generation approach and a Reynolds Averaged Navier-Stokes (RANS) flow-field solution method, thus forming an integrated aerodynamic design tool. A cost-effective Design Space Exploration (DSE) and optimisation strategy has been structured comprising methods for Design of Experiment (DOE), Response Surface Modelling (RSM), as well as genetic optimisation. The integrated framework has been deployed to optimise the aerodynamic performance of a separate-jet exhaust system for a large civil turbofan engine representative of future architectures. The optimisations carried out suggest the potential to increase the engine's net propulsive force compared to a baseline architecture, through optimum re-design of the exhaust system. Furthermore, the developed approach is shown to be able to identify and alleviate adverse flow-features that may deteriorate the aerodynamic behaviour of the exhaust system.

Keywords: Gas turbines, Turbofan, Exhaust nozzles, Aerodynamics, Computational Fluid Dynamics, Design Optimisation, Class-Shape Transformation functions

* Lecturer in Propulsion Integration, email: i.goulos@cranfield.ac.uk 


\section{NOMENCLATURE}

\section{Roman Symbols}

$\dot{m}$

$A_{\text {ratio }}$

$C_{\text {Bypass }}^{\text {Bys }}$

$C_{D}^{\text {Core }}$

$C_{V}^{O \text { verall }}$

$C_{D}^{\text {vent }}$

$F_{G}, F_{N}$

$h_{1}, h_{2}$

$L, R$

$l_{c r}^{c o w l}$

$l_{\text {vent }}^{\text {exit }}$

$M_{\infty}$

$M_{\text {vent }}^{\text {exit }}$

$N_{\text {Pearson }}$

$P, T$

$R_{p}^{2}$

$R_{C P}^{o f f s e t}$

$R_{\text {curve }}$

$R_{\text {fan }}$

$y_{b p}^{i n}$

$y_{b p}^{\text {out }}$

Greek Symbols

$\kappa_{C P}^{i n}$

$\kappa_{C P}^{o u t}$

$\kappa_{\text {len }}^{\text {in }}$

$\theta_{C P}^{\text {out }}$

$\theta_{c p}^{\text {plug }}$

$\theta_{c r}^{\text {cowl }}$

$\theta_{\text {nozzle }}^{\text {out }}$

Superscripts

() $)^{a m b}$

() $)^{\text {in/out }}$

() $)^{\text {inlet }}$

() Overall

\section{Subscripts}

()$_{0}$

()$_{b p}$

()$_{C P}$

()

() Exit

()$_{s t}$

() vent
Nozzle mass flow, $\mathrm{kg} / \mathrm{sec}$

Nozzle exit to charging plane area ratio, $=\frac{A_{C P}}{A_{\text {exit }}}$

Bypass exhaust nozzle discharge coefficient

Core exhaust nozzle discharge coefficient

Exhaust system overall velocity coefficient

Air-flow vent exhaust nozzle discharge coefficient

Gross and net propulsive force, $N$

Nozzle charging plane and exit plane height, $m$

Length and Radius, $m$

Non-dimensional core after-body (cowl) length, $=\frac{L_{c r}^{c o w l}}{R_{f a n}}$

Non-dimensional location of air-flow vent exhaust exit, $=\frac{L_{\text {vent }}^{\text {exit }}}{L_{c r}^{\text {cowl }}}$

Mach number (free-stream)

Air-flow vent exhaust exit Mach number

Pearson's product-moment of correlation

Pressure and Temperature, $P a$ and $K$

Coefficient of determination of $p$-th order

Charging plane radial offset relative to the nozzle exit plane, $m$

Curvature radius, $m$

Fan blade radius, $m$

Bypass duct normalised inner line radius, $=\frac{R_{b p}^{i n}}{L_{\text {duct }}^{i n}}$

Bypass duct normalised outer line radius, $=\frac{R_{b p}^{\text {out }}}{L_{d u c t}^{\text {in }}}$

Inner aeroline curvature radius ratio at the charging plane, $=\frac{R_{\text {curve }}^{\text {CPin }}}{h_{2}}$

Outer aeroline curvature radius ratio at the charging plane, $=\frac{R_{\text {curve }}^{C P \text { out }}}{h_{2}}$

Nozzle length ratio, $=\frac{L_{\text {in }}^{\text {Nozzle }}}{h_{2}}$

Outer aeroline slope at the charging plane, deg

Core plug after-body angle, deg

Core after-body (cowl) half-cone angle, deg

Nozzle outer line exit angle, deg

Referring to ambient conditions

Referring to the inner or outer nozzle aeroline, respectively

Referring to inlet conditions

Referring to the overall exhaust system

Referring to total flow conditions

Referring to the bypass exhaust nozzle

Referring to the nozzle charging plane

Referring to the core exhaust nozzle

Referring to the nozzle exit plane

Referring to static flow conditions

Referring to the air-flow vent exhaust nozzle 


\subsection{INTRODUCTION}

\subsection{Background}

Current trends in civil aviation dictate a continuing need to improve aircraft performance and reduce environmental impact. This necessitates, among others, the design and implementation of more fuel-efficient and environmentally friendly aircraft engines. Epstein ${ }^{(1)}$ noted that in order to conceptualise, design, and implement the next generation of civil turbofan engines, substantial improvements are required in the technologies used for the design of both cores and propulsors. Considering the envisaged core configurations, the dominant drive is towards the design of cores with increased Turbine Entry Temperature (TET) and Overall Pressure Ratio (OPR) to improve thermal efficiency $^{(2)}$. According to Guha ${ }^{(3)}$, future propulsor designs will employ higher By-Pass Ratios $\left(B P R=\frac{\dot{m}_{\text {bypass }}}{\dot{m}_{\text {core }}}\right)$ combined with lower Fan Pressure Ratios (FPR) to reduce specific thrust and improve propulsive efficiency. Indicatively, it is noted that future turbofan engines are expected to operate with a BPR of the order of $15+$ at Design Point (DP) mid-cruise condition, which is approximately $35 \%$ greater compared to contemporary civil aero-engines.

An increase in $B P R$ for a given value of net thrust $F_{N}$ results in greater engine mass flow $\dot{m}^{\text {inlet }}$ and consequently larger inlet momentum drag $F^{\text {inlet }}$. However, the associated gross propulsive force $F_{G}$ is also augmented accordingly which leads to a higher gross to net propulsive force ratio $\frac{F_{G}}{F_{N}}$. As an example, it is noted that the ratio $\frac{F_{G}}{F_{N}}$ changes from approximately 3 to 4 for increasing the value of $B P R$ from 11 to $15+$ at fixed values of OPR, TET, and $F_{N}$. Consequently, the net propulsive force $F_{N}$ and Specific Fuel Consumption (SFC) of future civil aeroengines are expected to be more sensitive to variations in gross propulsive force $F_{G}$ compared to contemporary architectures. Furthermore, the power-plant wetted area is also increased with BPR with a consequent impact on aspects related to airframe-engine integration and the associated installation aerodynamics ${ }^{(4,5)}$.

The aerodynamic behaviour of the exhaust system has a major impact on gross propulsive force $F_{G}{ }^{(6,7,8)}$. Hence, it is anticipated that the performance of the exhaust system will play a key role to the success of the next generation of civil aero-engines. Consequently, it is imperative that the associated design space is thoroughly explored and that the aerodynamic performance of the exhaust is optimised at an early stage of the power-plant design process.

\subsection{Aerodynamics of civil aero-engines with separate-jet exhausts}

Separate-jet exhaust systems are predominantly used in medium to high BPR civil turbofan aero-engines ${ }^{(9)}$. Figure 1 presents a notional axi-symmetric engine geometry equipped with separate-jet exhausts. Within the context of this work, the term "exhaust system" encompasses the bypass and core ducts and nozzles, as well as the components located downstream of each nozzle exit. The bypass nozzle after-body, also referred to as the "core after-body" or "core cowl", divides the bypass and core nozzle flows. An air-flow vent is usually located on the core after-body and is used to exhaust secondary air-flows. A protruding core plug is usually employed to reduce the core after-body length required to achieve a specified core nozzle exit area.

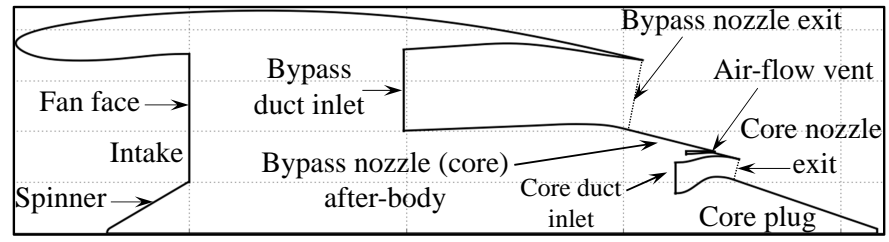

Figure 1. Notionally defined housing geometry for a turbofan engine equipped with separate-jet exhausts

The aerodynamic pressure and viscous forces exerted on the walls of the exhaust system can have a significant impact on the gross propulsive force $F_{G}$. According to Dusa et al. ${ }^{(10)}$, the reduction in $F_{G}$ due to non-isentropic flow conditions can reach approximately $1.5-2.0 \%$ relative to the case of ideal flow expansion to ambient static pressure. It is standard practice to quote the aerodynamic behaviour of an exhaust system relative to that of an ideal nozzle through the definition of the non-dimensional discharge and velocity coefficients, $C_{D}$ and $C_{V}$, respectively ${ }^{(11,12)}$. These essentially quantify the actual nozzle mass flow and resultant thrust, respectively, relative to the case of one-dimensional (1D) isentropic flow expansion to ambient static pressure ${ }^{(13)}$. The velocity coefficient $C_{V}$ is a quantitative measure of the thrust loss due to non-isentropic flow expansion. The associated loss mechanisms include the formation of shear layers between the freestream, bypass, and core jets, the skin friction exerted on the exhaust walls, as well as the manifestation of shock waves and expansion fans due to jet under-expansion. The discharge coefficient $C_{D}$ quantifies the reduction in nozzle mass flow due to flow blockage and momentum deficit associated with boundary layer development, as well as potential flow suppression due to external flow conditions. 
The advancement of Computational Fluid Dynamics (CFD) methods during the past decades has established it as a reliable tool for the prediction of aerodynamic flows in transonic exhaust systems ${ }^{(14,15)}$. Recently, Zhang $e t$ $a l .{ }^{(16,17)}$, through the first AIAA Propulsion Aerodynamics Workshop (PAW), demonstrated that for single-stream conical nozzles, the agreement between CFD predictions and experimental measurements in terms of $C_{D}$ and $C_{V}$ can reach approximately $0.2 \%$ and $0.5 \%$, respectively. However, Zhang et al. attributed these discrepancies primarily to the uncertainty of the experimental data, rather than physical accuracy of the employed CFD approach.

The second PAW workshop ${ }^{(18)}$ focused on the experimental and numerical investigation of the Dual Separate Flow Reference Nozzle (DSFRN), which is a separate-jet exhaust system representative of contemporary aeroengine designs. Experimental wind tunnel tests were carried out over a Fan Nozzle Pressure Ratio (FNPR) range from 1.4 to 2.6. It was found that, based on the average values predicted by workshop participants ${ }^{(19,20)}$, the axial thrust coefficient was calculated within a range of $0.6 \%$ of measured data ${ }^{(18)}$ for the investigated FNPR range.

Keith et al. ${ }^{(21)}$ described an integrated framework for the aerodynamic analysis of three-dimensional (3D) separate-jet exhaust systems for turbofan engines. Their numerical approach was based on Computational Fluid Dynamics (CFD) through the deployment of a Reynolds-Averaged Navier Stokes (RANS) scheme implemented in the CFL3D code ${ }^{(22)}$. Flow-field analyses were carried out and reported for two-dimensional (2D) axi-symmetric exhaust geometries as well as for 3D designs including the bifurcations and pylon. All investigated exhaust designs employed a simplified conical representation for the core after-body. Keith et al. concluded that the exhaust flow properties for the axi-symmetric cases are representative of those corresponding to the full 3D designs with respect to regions away from the influence of the bifurcations and pylon.

Clement et al. ${ }^{(23)}$ reported on the optimisation of the Low-Pressure (LP) exhaust system for a high-BPR turbofan engine. The employed geometric topology included the fan Outlet Guide Vanes (OGVs), the bypass duct, as well as structural components such as struts, fairing, and bifurcations. The bypass duct geometry was parametrised using second-order splines, whilst the 3D RANS flow-solver HYDRA ${ }^{(24)}$ was deployed to predict the aerodynamic behaviour of the combined exhaust system. A holistic optimisation strategy was devised including methods for DOE, surrogate modelling, and global optimisation. A random sequence generator ${ }^{(25)}$ was incorporated to sample the prescribed design space, whilst interpolation using Radial Basis Functions (RBF) ${ }^{(26)}$ was deployed to structure the required surrogate models. A Genetic Algorithm $(\mathrm{GA})^{(27)}$ was applied to optimise the exhaust design by minimising the total pressure loss in the bypass duct. The combined process was able to reduce the predicted total pressure loss within the duct by $0.1 \%$ relative to a baseline design.

\subsection{Scope of present work}

In light of the anticipated design trends outlined above, this paper presents a comprehensive approach for the DSE and optimisation of separate-jet exhaust systems for the next generation of civil aero-engines (Fig. 1). The proposed framework comprises a series of individual methods applicable to engine performance analysis, geometric parametrisation, aerodynamic analysis, DSE, and optimisation. Parametric design is carried out based on minimal user-input along with the flow-capacities established using a 0D engine analysis method ${ }^{(28)}$. A mathematical approach is developed using CST functions ${ }^{(29)}$ for the geometric design of axi-symmetric engine architectures with separate-jet exhausts. The developed approach inherits the intuitiveness and flexibility of Qin's aerofoil parametrisation method ${ }^{(30)}$ and extends its applicability to the design of exhaust ducts and nozzles.

The developed methodology is coupled with an automated mesh generation tool ${ }^{(31)}$ and a RANS flow-field solution method ${ }^{(32)}$. A computationally efficient DSE and optimisation strategy is formulated consisting of methods for $\mathrm{DOE}^{(33)}, \mathrm{RSM}^{(34)}$, as well as state-of-the-art genetic optimisation ${ }^{(35)}$. The combined approach is applied to explore the available design space and optimise the geometry of a separate-jet exhaust system for a Very-HighBypass-Ratio (VHBR) civil turbofan engine, representative of envisaged future architectures. High-order polynomial regression ${ }^{(36)}$ combined with Hinton visualisation $^{(37)}$ is employed to form sensitivity charts capable of identifying the dominant design variables that affect the aerodynamic performance of the exhaust. A comparative evaluation has been carried out between the optimum and datum exhaust geometries to assess the potential of the developed approach to automatically design optimum separate-jet exhaust systems for future civil aero-engines.

It is shown that the proposed method allows the aerodynamic design of separate-jet exhausts for a designated engine cycle, using only a limited set of intuitive design variables employed in standard industry practice. The optimisation carried out revealed significant potential to increase the engine's net propulsive force through optimum re-design of the employed exhaust system, compared to a notional baseline. Furthermore, the developed approach is shown to be able to identify and alleviate adverse flow-features that may deteriorate the aerodynamic behaviour of the exhaust system. Therefore, the proposed framework can be viewed as an enabler towards the design of optimally configured separate-jet exhausts, accompanied with increased net propulsive force and reduced SFC. 


\subsection{MATHEMATICAL MODEL DEVELOPMENT}

This work adapts the numerical approach developed by Goulos et al. ${ }^{(38,39)}$ for the aerodynamic analysis of civil gas-turbine aero-engines with separate-jet exhausts. The developed method has been named GEMINI (Geometric Engine Modeller Including Nozzle Installation). Figure 2 presents an upper-level illustration of the developed software architecture. GEMINI can automatically design, mesh, simulate, and optimise the geometry of an exhaust system based on a designated engine cycle and a limited set of key hard points prescribed by the user. GEMINI encompasses a series of fundamental modelling methods originally developed for; engine performance analysis ${ }^{(28)}$, exhaust duct and nozzle aeroline parameterisation ${ }^{(29,40,30,38)}$, RANS flow solution ${ }^{(31,32)}$, as well as DSE and MultiObjective Optimisation $(\mathrm{MOO})^{(39)}$. An analytical description of the individual modules has been provided by Goulos et al. ${ }^{(38,39)}$. Hence, only a brief synopsis of the system will be provided in this paper.

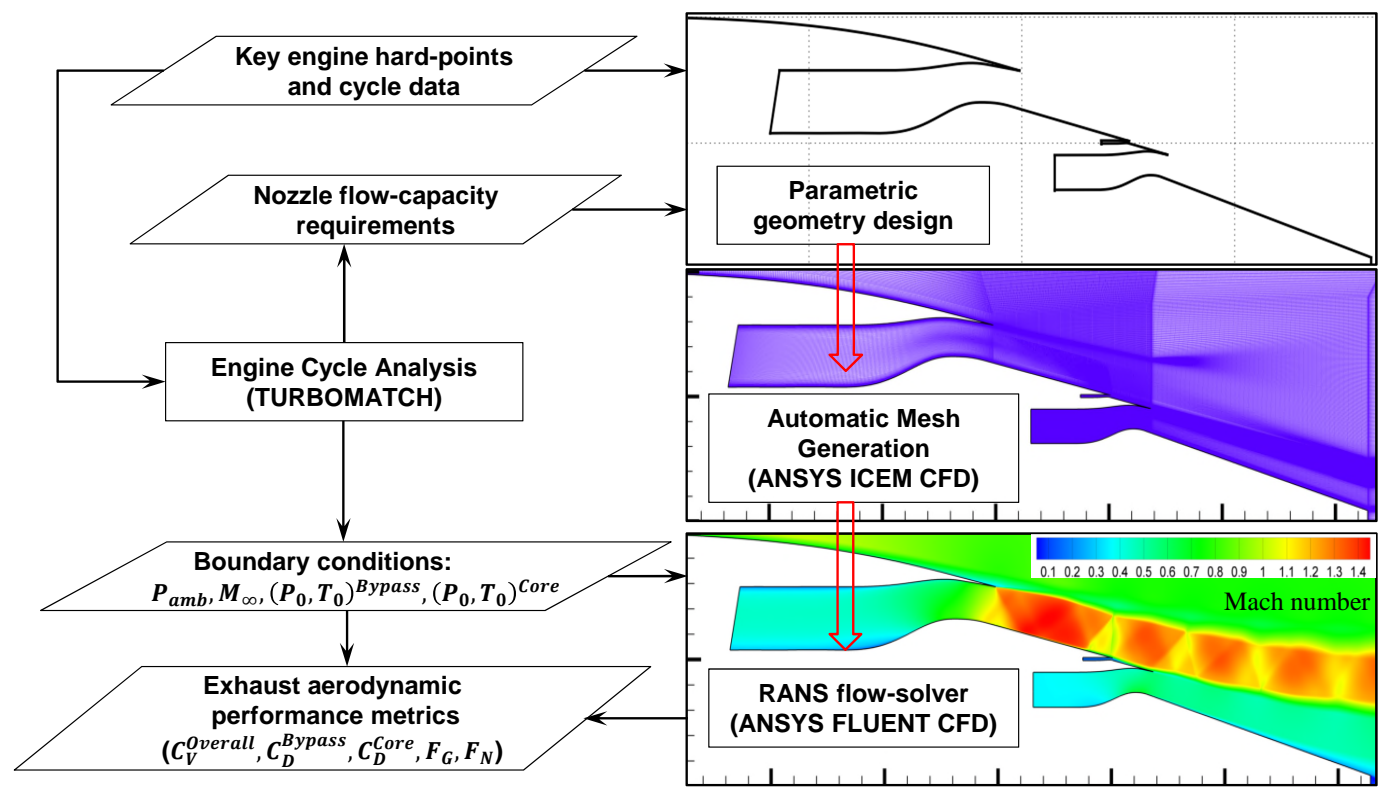

Figure 2. GEMINI: Upper-level overview software architecture

\subsection{GEMINI: Aerodynamic design and analysis of civil aero-engine exhaust systems}

The exhaust system design method in GEMINI is initialised by evaluating the aero-thermal behaviour of the engine for a series of user-defined operating points. This includes both Design Point (DP) as well as Off-Design (OD) conditions. Analysis is carried out using the OD approach method (TURBOMATCH) originally described by Macmillan ${ }^{(28)}$. The purpose of this process is two-fold: (a) it estimates the throat-area demand for the bypass and core exhaust nozzles, and (b) it determines the averaged flow properties at the inlet of each nozzle to be used as boundary condition in the aerodynamic analysis (Fig. 2). TURBOMATCH has been previously deployed in several studies in the literature for the prediction of DP, OD, as well as transient performance of gas turbine engines ${ }^{(41)}$.

Having established the required nozzle flow capacities, GEMINI derives the aerodynamic lines for the engine components such as the intake, nacelle, and exhaust system (Fig. 1). An automated mesh generation method is subsequently deployed to establish a multi-block structured grid $^{(31)}$ for the designed geometry. Thus, GEMINI establishes the computational domain upon which the viscous and compressible flow-field can be solved using a RANS flow-solution method ${ }^{(32)}$. The CFD methods and approach in GEMINI have been verified and validated by Goulos et al. ${ }^{(38)}$. Having obtained a converged flow solution, the numerical data are post-processed to determine the exhaust system's performance metrics. These include the bypass and core nozzle discharge coefficients, $C_{D}^{\text {Bypass }}$ and $C_{D}^{\text {Core }}$, respectively, as well as the overall exhaust velocity coefficient $C_{V}^{\text {Overall }}$ and gross propulsive force $F_{G}$. An overview of the aerodynamic metrics applicable to separate-jet exhausts has been provided by Goulos et al. ${ }^{(38)}$.

\subsection{Exhaust nozzle design and analysis}

GEMINI incorporates a parametric geometry definition based on the Class-Shape function Transformation (CST) method originally proposed by Kulfan ${ }^{(29,40)}$ and further developed by Qin ${ }^{(30)}$. The method ${ }^{(38)}$ inherits the intuitiveness and flexibility of Qin's CST variation ${ }^{(30)}$ and extends its applicability to the parametric representation of 
exhaust ducts and nozzles. The developed formulation expresses the bypass/core duct, nacelle exhaust, and afterbody aero-lines in completely closed form as functions of intuitive parameters. These have been selected based on standard practice in terms of exhaust design and include the following; nozzle Charging Plane (CP) to exit area ratio $A_{\text {ratio }}=\frac{A_{C P}}{A_{\text {exit }}}$, nozzle length ratio $k_{\text {len }}=\frac{L_{\text {nozzle }}}{h_{2}}$, aeroline curvature and slope at the nozzle CP location, $R_{\text {curve }}^{\text {in } / \text { out }}$ and $\theta_{C P}^{\text {out }}$, respectively, as well as nozzle outlet angles $\theta_{\text {in } / \text { out }}^{\text {nozle }}$. Figure 3(a) presents a notional nozzle geometry established parametrically using GEMINI. A detailed mathematical description of the employed design approach has been provided by Goulos et al. ${ }^{(38)}$.

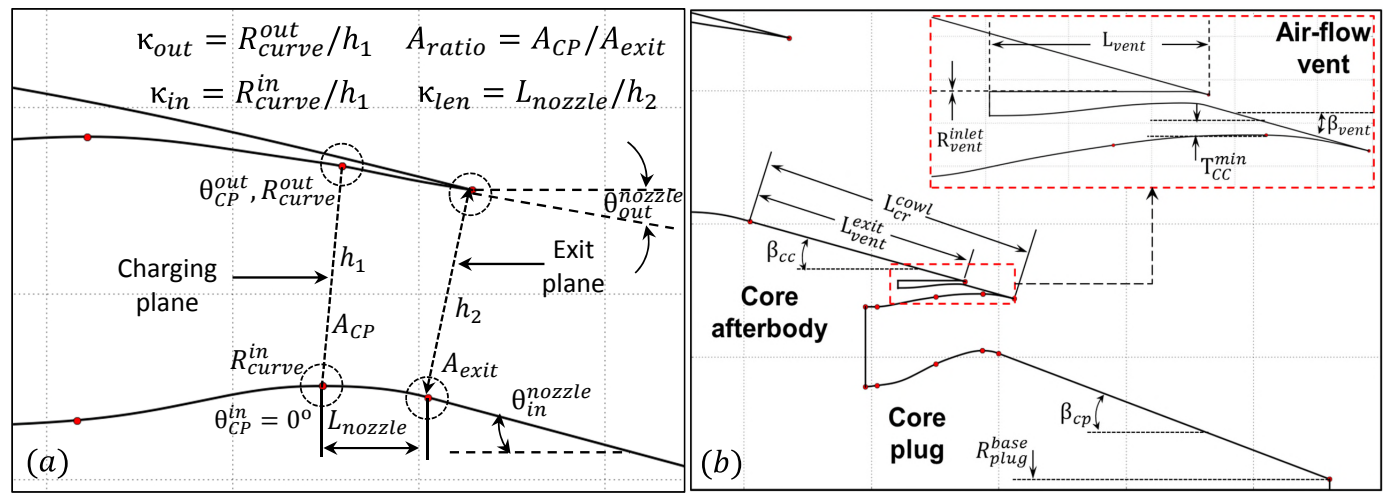

Figure 3. Parametric geometry definition of exhaust systems: (a) Exhaust nozzle. (b) Core after-body, air-flow vent, and protruding plug

The design of the exhaust nozzle downstream of the CP is initialised at the exit plane using the computed geometric area requirement. It is noted that for convergent nozzles, the geometric throat location is positioned at the exit plane. With respect to the design of convergent-divergent (con-di) nozzles, an effective con-di ratio is applied, effectively moving the throat location upstream relative to the designated exit plane. The rolling-ball area prediction method $^{(42)}$ is applied to the $\mathrm{CP}$ and throat, which results in a concise set of control points that reflect the constraints directly related to nozzle design parameters such as $A_{\text {ratio }}, k_{\text {len }}=\frac{L_{\text {nozzle }}}{h_{2}}, R_{\text {curve }}^{\text {in/out }}, \theta_{C P}^{\text {out }}$, and $\theta_{\text {in } / \text { out }}^{\text {nozle }}($ Fig. 3).

Furthermore, GEMINI incorporates a fully parametric geometry definition for the exhaust components located downstream of the bypass and core nozzle exits. The representation assumes a conical core after-body (cowl) with length $L_{c r}^{c o w l}$ and half-cone angle $\theta_{c r}^{c o w l}$. A protruding core plug with half-cone angle $\theta_{c r}^{\text {plug }}$ and base radius $R_{\text {plug }}^{\text {base }}$ is also employed as shown in Fig. 3(b). The geometric topology includes a parametric representation of the air-flow vent. This is essentially designed as a separate exhaust nozzle whose exit plane is located on the core after-body between the bypass and core nozzle exits. The geometry of the air-flow vent exhaust is also fully-parametric. Thus, the user is able to select its position $L_{v e n t}^{\text {exit }}$ on the core after-body and the duct length upstream of the vent exit $L_{v e n t}$.

\subsection{Design space exploration and optimisation}

GEMINI encompasses a cost-effective optimisation strategy that caters for the inherent non-linearity of transonic flow aerodynamics and reduces the computational overhead associated with multiple CFD evaluations ${ }^{(39)}$. The overall process has been reported by Goulos et al. ${ }^{(39)}$, thus only a brief synopsis will be provided in this paper.

The analysis environment of GEMINI comprises modules for Design Space Exploration (DSE), Response Surface Modelling (RSM - also referred to as surrogate modelling), parameter identification, and MOO. The DSE method comprises two parts; (a) an initial Design of Experiment (DOE) which strategically populates the design space, and (b) the formulation of RSMs using the DOE sample data. A DOE is a systematic approach to get the maximum amount of information out of a given sample. The Latin Hypercube Design (LHD) algorithm ${ }^{(43)}$ has been selected for this work. Having completed the computational process driven by the LHD DOE, RSMs can be subsequently structured using the sample data as model inputs. Interpolation using Gaussian Processes Regression $^{(34)}$ (Kriging Interpolation) is the method of choice for this work.

The derived RSMs can be used subsequently to predict the aerodynamic behaviour of new exhaust system geometries. GEMINI incorporates RSMs as drivers during the optimisation process instead of relying directly on CFD analysis. The underlying purpose is to mitigate the excessive computational overhead associated with numerous CFD evaluations. The classical Leave-One-Out (LOO) cross-validation method ${ }^{(44)}$ is deployed to evaluate the predictive accuracy of the structured RSMs prior to utilising them in an automated design optimisation environment. After successful approximation of the system's response to geometric inputs, the available design space can 
be systematically explored for potentially optimum exhaust designs. The selected optimisation method has to be immune to the danger of being trapped within design space regions containing locally optimum solutions. Hence, the deployment of a global method is essential. The Non-dominated Sorting Genetic Algorithm II (NSGA-II) originally proposed by Deb et al. ${ }^{(35)}$ has been selected to carry out the optimisations reported in this paper.

\subsection{RESULTS AND DISCUSSION}

The proposed framework (GEMINI) has been deployed to optimise the aerodynamic design of the LP exhaust system and core after-body aerolines for a VHBR civil aero-engine. The baseline power-plant architecture was defined to be representative of future large turbofans and the engine cycle was compiled using publicly available information $^{(45)}$. The axi-symmetric geometry for the datum exhaust system is illustrated in Fig. 1(a). Aerodynamic analyses have been carried-out at DP mid-cruise conditions $\left(M_{\infty}=0.85\right.$, Alt. $\left.=10668 m\right)$. The employed boundary conditions in terms of bypass and core nozzle pressure ratios have been documented by Goulos et al. ${ }^{(38,39)}$. The associated flow solution for the baseline engine exhaust design is illustrated graphically in Fig. 4. It can be observed that for cruising flight, the bypass exhaust nozzle is choked. However, due to the lower values of nozzle pressure ratio $^{(38)}$, the core nozzle is un-choked during mid-cruise conditions.

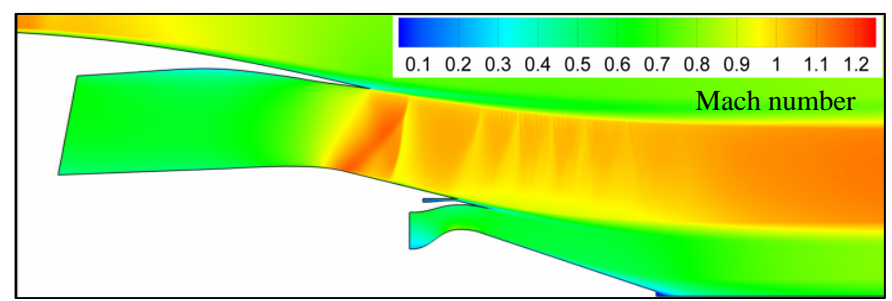

Figure 4. Mach number variation for the baseline exhaust system design at DP mid-cruise conditions

\subsection{Definition of investigated design space}

Figure 5 demonstrates the design variables used to establish a parametric representation of the investigated separate-jet exhaust system. A total of 12 design variables are employed to establish an analytical geometry definition for the LP exhaust and core after-body aerolines of interest. The overall design space comprises parameters that directly control the geometry of the bypass duct $\left(y_{b p}^{\text {in }}\right.$ and $\left.y_{b p}^{\text {out }}\right)$, the bypass nozzle $\left(A_{\text {ratio }}, \kappa_{l e n}^{\text {in }}, \theta_{C P}^{\text {out }}, \theta_{\text {nozzle }}^{\text {out }}, \kappa_{C P}^{\text {in }}\right.$, and $\left.\kappa_{C P}^{\text {out }}\right)$, core after-body $\left(l_{c r}^{\text {cowl }}\right.$ and $\left.\theta_{C R}^{\text {cowl }}\right)$, and air-flow vent $\left(M_{\text {vent }}^{\text {exit }}\right.$ and $\left.l_{\text {vent }}^{\text {exit }}\right)$. Figure 5 shows that the employed parametric geometry definition can represent a wide range of exhaust geometries, thus ensuring sufficient diversity in the design space. The mathematical definition of each variable is also provided in Fig. 5 for consistency.

\subsection{Design space exploration and parameter identification}

GEMINI was deployed to assess the aerodynamic behaviour of the investigated exhaust system throughout the specified domain. The available design space was discretised with the deployment of the LHD method ${ }^{(43)}$. A database containing approximately 720 exhaust geometries was compiled. Prior to commencing with the optimisation process, the obtained CFD database was analysed to evaluate the general response of the design space and to identify the dominant geometric parameters that influence the aerodynamic performance of the exhaust system. A series of well-established correlation methods such as those of Pearson ${ }^{(46)}$ and Spearman ${ }^{(47)}$, as well as high-order polynomial regression $^{(36)}$ were employed to highlight any linear and non-linear correlations between the imposed design variables and associated aerodynamic metrics. Polynomial regression was found to be the most appropriate method for the specific application due to its flexibility in defining the order of correlation.

Figures 6(a) and (b) present an example of polynomial regression applied to estimate the influence of normalised core cowl length $l_{c r}^{c o w l}$ (Fig. 5(f)) on the behaviour of the investigated design space. The parameter of interest $\left(l_{c r}^{c o w l}\right)$ is correlated against $C_{V}^{\text {Overall }}$ and $C_{D}^{\text {Core }}$ in Figs. 6(a) and (b), respectively. The individual symbols correspond to CFD data, whilst the solid lines show the behaviour of polynomial expressions fitted through the data. Analysis is carried out using up to $5^{\text {th }}$-order polynomial functions $(p=5)$. The calculated coefficients of determination $\left(R_{p}^{2}\right)$ are also reported for each order $p$ in Figs. 6(a) and (b) for $C_{V}^{\text {Overall }}$ and $C_{D}^{\text {Core }}$, respectively. The computed $R_{p}^{2}$ values indicate the average proximity of the regression lines to the fitted CFD data and can range between zero and unity.

It can be observed that both $C_{V}^{\text {Overall }}$ and $C_{D}^{\text {Core }}$ follow a monotonically ascending trend with increasing $l_{c r}^{\text {cowl }}$ considering the initial $30 \%$ of the examined variable range. This corresponds to roughly $10 \%$ in terms of core 

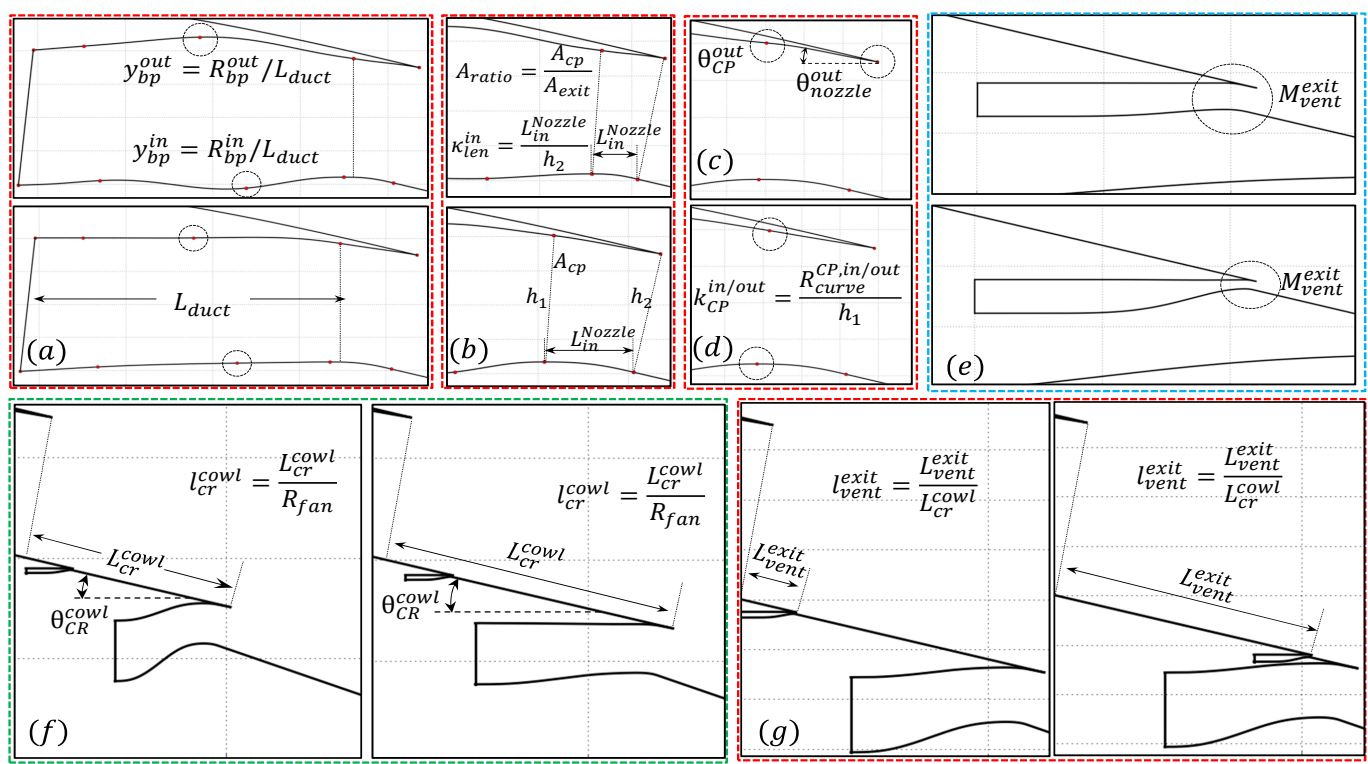

Figure 5. Illustrative description of design variables: (a) Bypass duct inner and outer line position $y_{b p}^{\text {in/out }}$, (b) Nozzle CP to exit area ratio $A_{\text {ratio }}$ and length ratio $\kappa_{l e n}^{\text {in }}$, (c) Outer line slope at the $\mathrm{CP} \theta_{C P}^{\text {out }}$ and nozzle exit $\theta_{n o z z l e}^{\text {out }}$, (d) CP inner/outer curvature radius ratio $\kappa_{C P}^{\text {in/out }}$, (e) Air-flow vent exit Mach no. $M_{\text {vent }}^{\text {exit }}$, (f) Core after-body (cowl) length $l_{c r}^{\text {cowl }}$ and half-cone angle $\theta_{C R}^{\text {cowl }}$, (g) Air-flow vent exit position $l_{\text {vent }}^{\text {exit }}$

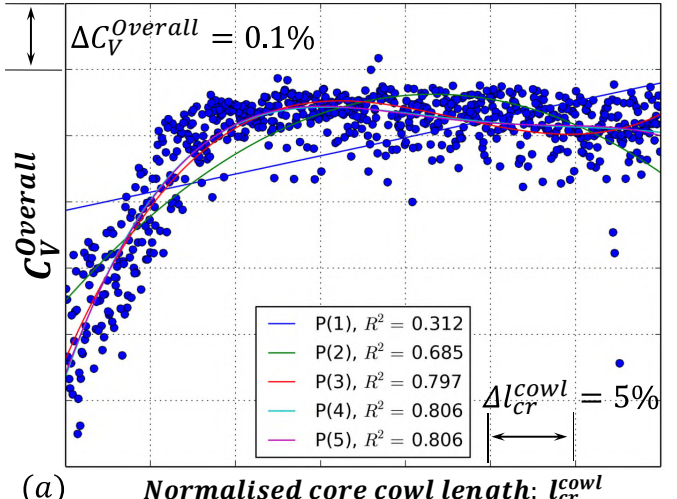

(a)

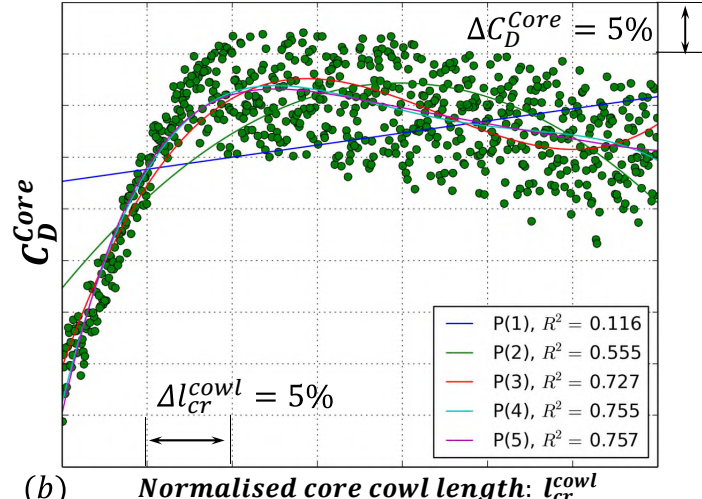

(b)

Normalised core cowl length: $l_{c r}^{\text {cow }}$

Figure 6. Polynomial regression analysis: (a) correlation between $l_{c r}^{\text {cowl }}$ and $C_{V}^{\text {Overall }}$, (b) correlation between $l_{c r}^{\text {cowl }}$ and $C_{D}^{\text {Core }}$

after-body length increase relative to the lower bound. The associated changes in aerodynamic performance concerning $C_{V}^{\text {Overall }}$ and $C_{D}^{\text {Core }}$ reach approximately $0.55 \%$ and $40 \%$ of the ideal levels, respectively. The general trend noted subsequently in the data suggests a small but gradual reduction in both $C_{V}^{\text {Overall }}$ and $C_{D}^{\text {Core }}$ which amounts to approximately $0.05 \%$ and $5 \%$, respectively, relative to the corresponding peak values.

Figures 6(a) and (b) show that $C_{V}^{\text {Overall }}$ and $C_{D}^{\text {Core }}$ respond similarly to changes in $l_{c r}^{\text {cowl }}$. This indicates that both performance metrics are affected by the same flow phenomena. The poor aerodynamic performance noted for very low values of $l_{c r}^{\text {cowl }}$ is associated with highly-aggressive core duct aerolines that lead to separated flow regions near the core nozzle $\mathrm{CP}$ and plug. This results in a severe reduction of core nozzle mass flow $\left(C_{D}^{\text {Core }}\right)$ and thrust $\left(C_{V}^{\text {Overall }}\right)$ relative to the baseline exhaust design. The aforementioned adverse flow-mechanisms are mitigated with increasing $l_{c r}^{c o w l}$. However, for large values of $l_{c r}^{\text {cowl }}$ the core nozzle becomes excessively long which leads to increased total pressure losses due to internal skin friction as well as flow separation on the core after-body. Hence, a gradual reduction in both $C_{V}^{\text {Overall }}$ and $C_{D}^{\text {Core }}$ is also noted for large values of $l_{c r}^{\text {cowl }}$.

The aforementioned aerodynamic behaviour is highly non-linear and is described quantitatively in terms of $C_{V}^{\text {Overall }}$ and $C_{D}^{\text {Core }}$ in Figs. 6(a) and (b), respectively. Due to the non-linearity of the investigated system, the classical concept of principal correlation based on linear regression cannot capture the response of the design space in an adequate manner. Figure $6(\mathrm{~b})$ shows that the coefficient of determination $\left(R_{p}^{2}\right)$ calculated using linear regression $(p=1)$ is of the order of $0.116\left(R_{p=1}^{2}=0.116\right)$. This indicates no apparent correlation between $l_{c r}^{\text {cowl }}$ and $C_{D}^{\text {Core }}$. 
A relatively low linear correlation coefficient is also identified for $C_{V}^{\text {Overall }}$ in Fig. 6(a) $\left(R_{p=1}^{2}=0.312\right)$. However, increasing the order of polynomial regression results in a dramatic change in $R_{p}^{2}$. Specifically, the coefficient of determination relating $l_{c r}^{\text {cowl }}$ to $C_{V}^{\text {Overall }}$ rises from 0.312 to 0.806 when increasing $p$ from 1 to $5\left(R_{p=5}^{2}=0.806\right)$. A similar trend is observed for $C_{D}^{\text {Core }}$ with $R_{p=5}^{2}=0.757$. A graphical representation of this behaviour is shown in Figs 6(a) and (b) with the associated regression lines being able to better fit the CFD data with increasing order of regression $p$. The observed behaviour highlights the necessity for using higher-order regression methods when analysing the aerodynamic behaviour of separate-jet exhaust systems in an automated DSE environment.

The aerodynamic interdependency between $C_{V}^{\text {Overall }}$ and $C_{D}^{\text {Core }}$ is shown in Fig. 7(a) where the polynomial regression analysis is applied to estimate the correlation between the two metrics. It can be observed that the predicted interrelationship exhibits a noticeable element of linearity. The estimated coefficient of determination when using linear regression is $R_{p=1}^{2}=0.673$ and is relatively independent of the regression order $p$ as shown in Fig. 7(a).
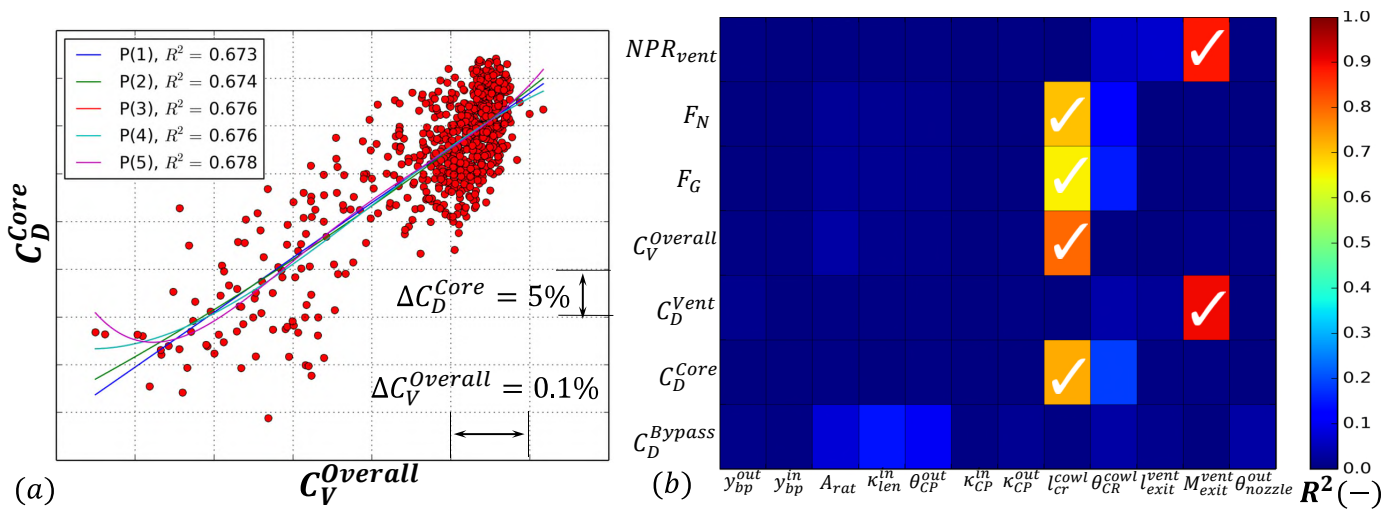

Figure 7. Polynomial regression analysis: (a) correlation process between $C_{V}^{\text {Overall }}$ and $C_{D}^{\text {Core }}$, (b) Hinton visualisation of $5^{\text {th }}$-order polynomial regression applied throughout entire design space

Figure 7(b) illustrates the system response correlation matrix derived through systematic exploration of the design space using $5^{\text {th }}$-order polynomial regression. The results are presented in the form of Hinton visualisation. Hinton diagrams can be useful in visualising numerical data in linear algebra, particularly considering weighting or correlation matrices ${ }^{(37)}$. The presented matrix demonstrates the distribution between the explored design variables (Fig. 5) and the associated coefficients of determination $R_{p=5}^{2}$ that relate them to the design outputs of interest.

The results depicted in Fig. 7(b) clearly show that both $C_{D}^{\text {Core }}$ and $C_{V}^{\text {Overall }}$ are primarily affected by the core afterbody length $\left(l_{c r}^{c o w l}\right)$. In other words, $l_{c r}^{\text {cowl }}$ is a dominant design parameter in terms of its impact on the aerodynamic performance of the exhaust system. Furthermore, Fig. 7(b) shows that the dominant design variables that influence $C_{D}^{\text {Bypass }}$ are the nozzle length ratio $\kappa_{\text {len }}^{\text {in }}$ (Fig. 5(b)) and the outer aeroline slope at the CP $\theta_{C P}^{\text {out }}$ (Fig. 5(c)). The aerodynamic impact of the air-flow vent exit Mach number $M_{v e n t}^{\text {exit }}$ on its total to static pressure ratio $N P R_{\text {vent }}=\frac{P_{0}^{\text {inlet }}}{P_{s t^{\text {tamb }}}}$ and its discharge coefficient $C_{D}^{\text {Vent }}$ are also readily apparent. It is noted that the air-flow vent is modelled as a prescribed mass-flow inlet. Therefore, for a fixed inlet mass-flow, the required $P_{0}$ at the vent entry is directly dependent on the vent throat area which is uniquely defined by $M_{v e n t}^{\text {exit }}$. This establishes a linear dependency for $N P R_{\text {vent }}$ and $C_{D}^{\text {Vent }}$ on $M_{\text {vent }}^{\text {exit }}$.

Therefore, it has been shown that the use of high-order polynomial regression combined with Hinton visualisation can constitute a useful tool in the holistic representation of complex aerodynamic systems. Furthermore, the proposed method enables the rapid identification of dominant design variables and provides insight on the underlying mechanisms that govern the aerodynamic response of the exhaust system. Thus, the proposed method can be considered as an indispensable DSE tool that can provide insight and guidance to analysts prior to optimisation.

\subsection{Surrogate modelling and cross-validation}

Having compiled a comprehensive exhaust design data-base for the investigated VHBR engine architecture, the obtained aerodynamic results were utilised to formulate surrogate models (RSMs) that can approximate the response of the design space with sufficient accuracy. The approach employed in this paper was based on interpolation using Gaussian Processes Regression ${ }^{(34)}$. The incorporated Kriging interpolation model implementation utilised a quadratic regression function combined with absolute exponential auto-correlation. 

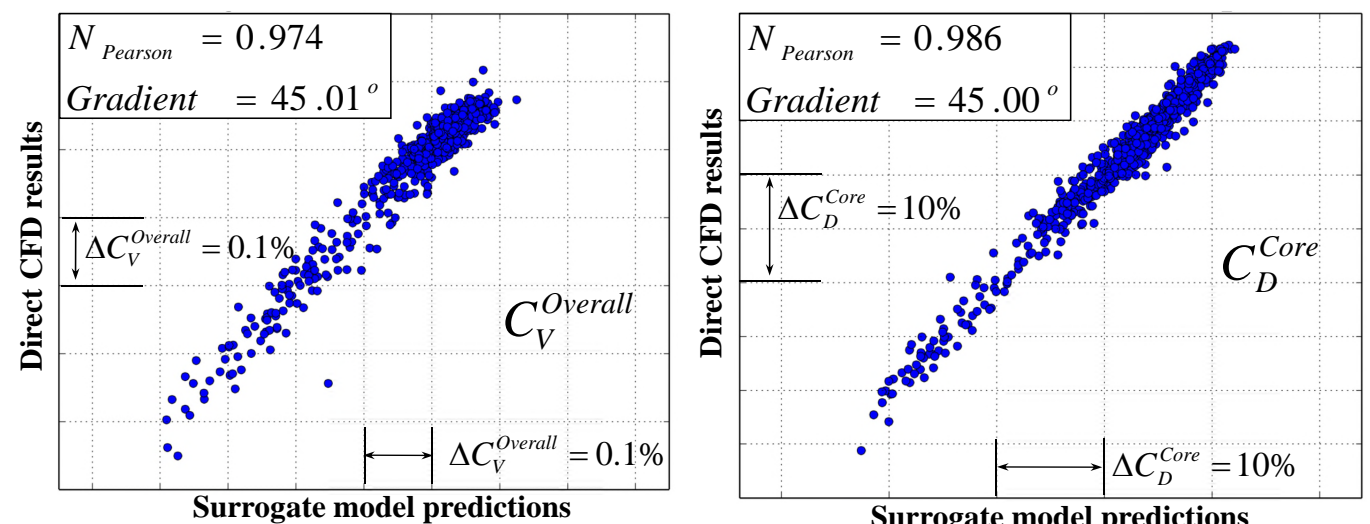

Surrogate model predictions

Figure 8. LOO cross-validation applied to the structured surrogate models for for: (a) $C_{V}^{\text {Overall }}$ and (b) $C_{D}^{\text {Core }}$

The well-established LOO cross-validation method ${ }^{(39)}$ was employed to evaluate the quality of the RSMs. The method is applied as follows: An RSM is created for each of the DOE sample designs so that an RSM is uniquely associated with a specific sample-point. The data used to formulate each RSM include the entire range of LHD DOE data with the exception of its corresponding sample-point. Subsequently, the sample-point left-out of the data-base is compared against predictions made with its associated RSM. This process is repeated for all samples. The obtained RSM predictions are then cross-correlated against the original DOE results in terms of Pearson's product moment of correlation $N_{\text {Pearson }}{ }^{(46)}$ along with the gradient of the associated linear regression line.

This process is illustrated in Figs. 8(a) and (b) for the $C_{V}^{\text {Overall }}$ and $C_{D}^{\text {Core }}$, respectively. It is noted that a perfectly linear correlation corresponds to $N_{\text {Pearson }}=1$ and a regression line gradient of $45^{\circ}$. It can be observed that the computed values of $N_{\text {Pearson }}$ when correlating RSM predictions with direct CFD results are of the order of 0.974 and 0.986 for $C_{V}^{\text {Overall }}$ and $C_{D}^{\text {Core }}$, respectively. Furthermore, the associated gradients of the calculated linear regression lines are almost exactly $45^{\circ}$ considering both performance metrics of interest. The computed quality metrics indicate the excellent predictive accuracy of the formulated RSMs.

\subsection{Exhaust system design optimisation}

Having extensively evaluated and gained confidence in the predictive accuracy of the structured RSMs, they can be deployed as drivers in an automated design optimisation process. The key advantage of using RSMs stems from the minuscule computational time requirement compared to using direct CFD evaluations. This enables the derivation of optimum designs for various combinations of objective functions in almost real-time. The objective of the optimisation is to identify an exhaust geometry that maximises the aerodynamic performance of the exhaust system. The NSGA-II method ${ }^{(35)}$ was employed for all optimisations reported in this paper.

The optimisation was constrained by imposing appropriate bounds to the design variables shown in Fig. 5. The overall velocity coefficient $C_{V}^{\text {Overall }}$ was selected as the objective function to be maximised. This is because, due to the nature of modelling assumptions used in the current $\mathrm{CFD}$ approach, $C_{V}^{\text {Overall }}$ was found to be the most objective metric to quantify aerodynamic performance ${ }^{(38)}$. The population size was set to 20 times the number of variables which resulted in 240 designs per generation. A convergence criterion of $10^{-15}$ was applied on the average consecutive mutations per generation. A maximum limit of 200 generations was imposed in the evolutionary iteration. However, the defined convergence criterion was satisfied well within the maximum number of generations.

The aerodynamic flow solutions obtained for the datum and optimised exhausts are illustrated in Figs. 9(a) and (b), respectively. Results are presented in terms of Mach number variations within the bypass and core ducts and nozzles, as well as over the core after-body and plug. The relative alterations in the design parameters between the two geometries are also depicted for consistency. The aerodynamic analysis of the datum design (Fig. 9(a)) reveals the existence of a strong normal shock on the core after-body. As a result, the bypass jet's total pressure and gauge stream force are reduced, thus deteriorating the aerodynamic performance of the exhaust system. Furthermore, it can be observed that the datum exhaust geometry employs a relatively short core after-body $\left(l_{c r}^{\text {cowl }}\right)$ and consequently a short core nozzle with an aggressive inner aeroline. As a result of the aggressive radial flow-turning, the inner core line induces an adverse pressure gradient that propagates upstream near the vicinity of the core nozzle inlet.

The aerodynamic behaviour of the optimised exhaust system presented in Fig. 9(b) demonstrates that the optimisation has successfully mitigated the adverse flow-features present in the datum exhaust. It can be observed that the optimised exhaust system employs a notably longer core-after body $l_{c r}^{\text {cowl }}$ with a lower half-cone angle $\theta_{c r}^{\text {cowl }}$ 

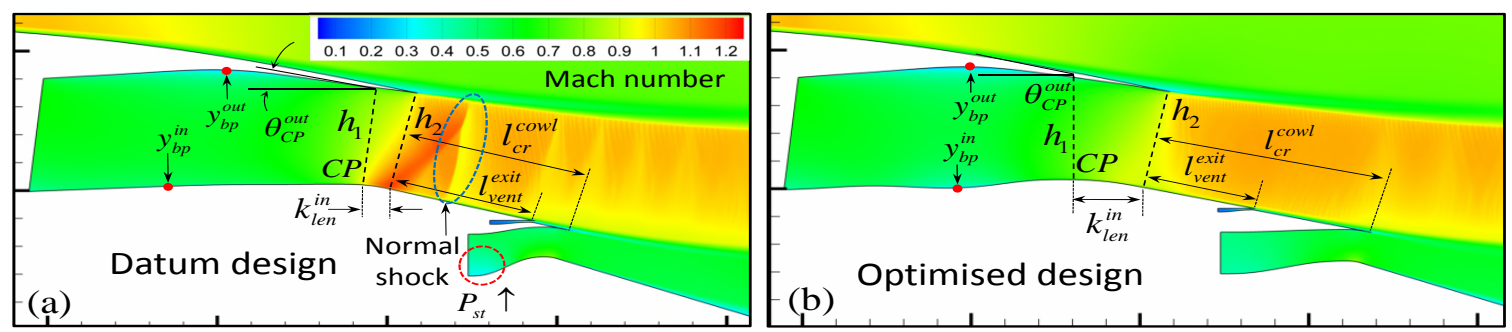

Figure 9. Aerodynamic comparison between initial and final exhaust system designs: (a) datum exhaust geometry and (b) exhaust geometry optimised for $C_{V}^{\text {Overall }}$

resulting in an elongated core nozzle. This geometric modification has lessened the impact of the adverse pressure gradients associated with the aggressiveness of the inner core aeroline of the datum exhaust. In addition to the above, the optimised exhaust system incorporates a bypass duct geometry that gradually diffuses the inlet flow upstream of the bypass nozzle CP. This is done to maintain low velocities and reduce skin friction losses in the duct. The bypass duct geometry subsequently converges to the nozzle $\mathrm{CP}$ before entering the bypass nozzle where it is further accelerated to sonic conditions at the nozzle throat.

Furthermore, the strong normal shock previously noted on the core after-body of the datum geometry has been alleviated and the transonic flow-topology aft of the bypass nozzle exit is free of any notable adverse flow features. As a result, the bypass flow expansion for the optimised design is closer to the ideal isentropic process compared to that achieved by the datum exhaust. This has been accomplished by increasing the nozzle length ratio $\kappa_{\text {len }}^{\text {in }}$ (Fig. 5(b)) and relaxing the inner line curvature distribution upstream of the nozzle exit. This design adjustment allows the bypass flow to align with the core after-body in a more gradual manner before expanding to ambient conditions. As a consequence, the flow acceleration induced by the inner line surface curvature is significantly reduced. This effectively lowers the local maximum Mach number downstream of the nozzle exit which consequently mitigates the adverse shock topology on the core after-body as shown in Fig. 9(b). Therefore, it has been shown that the approach described in this paper is able to identify and alleviate unfavourable flow-features that may affect the aerodynamic performance of a separate-jet exhaust system in an adverse manner.

The combined design adjustments showcased in Fig. 9(b) have resulted in an aerodynamic performance improvement of the order of $0.3 \%$ and $0.065 \%$ in terms of $C_{D}^{\text {Bypass }}$ and $C_{V}^{\text {Overall }}$, respectively, relative to the datum exhaust design (Fig. 9(a)). Furthermore, for this example, the estimated improvement in $C_{D}^{\text {Core }}$ reaches approximately $2 \%$ which indicates once again its dependency on $C_{V}^{\text {Overall }}$ as demonstrated in Fig. 7(b). Hence, it can be concluded that the developed methodology has been successful in synthesising an exhaust configuration with notable aerodynamic performance improvement relative to a datum exhaust system.

\subsection{CONCLUSIONS}

This paper has presented an integrated framework which targets the aerodynamic analysis and optimisation of separate-jet exhaust systems for the next generation of civil aero-engines. A mathematical approach has been developed based on CST functions for the parametric representation of exhaust system components such as annular ducts, nozzles, after-bodies, and plugs. The proposed parametric geometry definition has been coupled with an automated RANS CFD modelling approach, thus formulating a standalone aerodynamic tool for exhaust system design and analysis. A computationally-efficient DSE and optimisation strategy has been adapted comprising methods for DOE, hyper-space correlation, surrogate modelling, as well as state-of-the-art genetic optimisation. The combined approach has been deployed to examine the design space governing the aerodynamic behaviour of the exhaust system for a VHBR turbofan engine with anticipated entry to service by the year 2025 .

The methodology proposed in this paper has been successful in synthesising an engine geometry with substantially improved aerodynamic performance through optimum re-design of the incorporated exhaust system. It has been shown that high-order polynomial regression combined with Hinton visualisation can rapidly identify the dominant design parameters and physical mechanisms that govern the aerodynamic behaviour of the exhaust system. Furthermore, it has been demonstrated that the proposed approach can alleviate adverse flow-phenomena that may deteriorate the aerodynamic performance of the exhaust system. Hence, the methodology described in this paper constitutes a useful tool for the conceptual design of optimum exhaust geometries that provide increased net propulsive force and consequently reduced SFC. 


\section{ACKNOWLEDGEMENTS}

This project was partially funded by Innovate UK.

\section{REFERENCES}

1. Epstein, A. H., "Aeropropulsion for Commercial Aviation in the Twenty-First Century and Research Directions Needed," AIAA Journal, Vol. 52, (5), May 2014, pp. 901-911.

2. Walsh, P. and Fletcher, P., Gas Turbine Performance Engineering, Blackwell Publishing, 2004.

3. Guha, A., "Optimum Fan Pressure Ratio for Bypass Engines with Separate or Mixed Exhaust Streams," AIAA Journal of Propulsion and Power, Vol. 17, (5), September-October 2001 2001, pp. 1117-1122.

4. Stankowski, T., MacManus, D. G., Sheaf, C. T., and Christie, R., "Aerodynamics of Aero-Engine Installation," Proceedings of the Institution of Mechanical Engineers, Part G: Journal of Aerospace Engineering, Vol. 230, (14), February 2016, pp. 2673-2692.

5. Stankowski, T., MacManus, D. G., Robinson, M., and Sheaf, C. T., "The Aerodynamic Effects of a VHBR Engine Installation to the Common Research Model," Journal of Aircraft, Vol. (Accepted for publication - to appear), 2016.

6. MIDA, S. G., "Guide to In-Flight Thrust Measurement of Turbojets and Fan Engines," Advisory Group for Aerospace Research and Development, AGARD-AG-237, 7 Rue Ancelle 92200 Newilly, Sur Seine, France, January 1979.

7. AGARD, "Aerodynamics of Power Plan Installation," Advisory Group for Aerospace Research and Development, AGARD-CP-301, 7 Rue Ancelle 92200 Newilly, Sur Seine, France, May 1981.

8. Covert, E. E., James, C. R., Kimsey, W. M., Rickey, G. K., and Rooney, E., Thrust and Drag: Its Prediction and Verification (Progress in Astronautics and Aeronautics Series), American Institute of Aeronautics \& Astronautics, Reston, VA, 1985.

9. Rolls-Royce, The jet engine, Wiley-Blackwell, Hoboken, New Jersey, United States, 5th ed., 1966.

10. Dusa, D., Lahti, D., and Berry, D., "Investigation of Subsonic Nacelle Performance Improvement Concept," 18th Joint Propulsion Conference, Cleveland, OH, U.S.A, June 21-23 1982.

11. Wilson, E. A., Adler, D., and Bar-Yoseph, P., “Nozzle Performance Modeling,” AIAA Journal, Vol. 40, (7), July 2002, pp. $1331-1338$.

12. Bussman, W. and White, J., "Explicit Equation for Flow Through American Society of Mechanical Engineers Nozzle Meters," AIAA Journal, Vol. Vol. 36, (9), September 1998, pp. 1744-1746.

13. Decher, R. and Tegeler, D. C., "High Accuracy Force Accounting Procedures for Turbo Powered Simulator Testing," 11th AIAA Joint Propulsion Conference, AIAA-1975-1324, Anaheim, CA, U.S.A, 1975.

14. Sloan, B., Wang, J., Spence, S., Raghunathan, S., and Riordan, D., "Aerodynamic Performance of a Bypass Engine with Fan Nozzle Exit Area Change by Warped Chevrons," IMechE Journal of Aerospace Engineering, Vol. 224, (6), January 2010, pp. 731-743.

15. Malecki, R. E. and Lord, K., "Aerodynamic Performance of Exhaust Nozzles Derived from CFD Simulation,” 31st AIAA Joint Propulsion Conference, AIAA-1995-2623, San Diego, CA, U.S.A, 1995.

16. Zhang, Y., Chen, H., Zhang, M., Zhang, M., Li, Z., and Fu, S., "Performance Prediction of Conical Nozzle Using Navier-Stokes Computation," AIAA Journal of Propulsion and Power, Vol. 31, (1), January-February 2015, pp. 192-203.

17. Zhang, Y., Chen, H., Fu, S., Zhang, M., and Zhang, M., "Drag Prediction Method of Powered-On Civil Aircraft Based on Thrust-Drag Bookkeping,” Chinese Journal of Aeronautics, Vol. 28, (4), August 2015, pp. 1023-1033.

18. Mikkelsen, K. L., Myren, D. J., Dahl, D. G., and Christiansen, M. D., "Initial Subscale Performance Measurements of the AIAA Dual Separate Flow Reference (DSFR) Nozzle," 51st AIAA/SAE/ASEE Joint Propulsion Conference, No. AIAA Paper No. 2015-3883, Orlando, Florida, USA, 2015.

19. Domel, N. D., "Perspectives on Propulsion CFD for Nozzle Applications Relevant to the AIAA Propulsion Aerodynamics Workshop," 51st AIAA/SAE/ASEE Joint Propulsion Conference, No. AIAA Paper 2015-3778, Orlando, Florida, USA, 2015.

20. Li, Z., Che, H., and Zhang, M., "NSAWET Results of the Dual Separate Flow Reference Nozzle From AIAA PAW02," 51st AIAA/SAE/ASEE Joint Propulsion Conference, No. AIAA Paper No. 2015-3779, Orlando, Florida, USA, 2015.

21. Keith, B. D., Uenishi, K., and Dietrich, D. A., "CFD-Based Three-Dimensional Turbofan Exhaust Nozzle Analysis System," AIAA Journal of Propulsion and Power, Vol. 9, (6), November-December 1993, pp. 840-846.

22. Anderson, W. K., Thomas, J. L., and Whitfield, D. L., "Multigrid Acceleration of the Flux-Split Euler Equations," AIAA Journal, Vol. 26 , (6), June 1988, pp. 649-654.

23. Clemen, C., Albrecht, P., and Herzog, S., "Systematic Optimisation of a Turbofan Bypass Duct System,” ASME Turbo Expo 2012: Turbine Technical Conference and Exposition, No. GT2012-68276, Copenhagen, Denmark, June 11-15 2012.

24. Lapworth, B. L., "HYDRA-CFD: A Framework for Collaborative CFD Development," International Conference on Scientific and Engineering Computation (IC-SEC), Singapore, June 30-July 022004.

25. Sobol, I. M., "On the Systematic Search in a Hypercube," Siam J. Numer. Anal., Vol. 16, (5), October 1979.

26. Wright, G. B., Radial Basis Function Interpolation: Numerical and Analytical Development, Ph.D. thesis, Department of Applied Mathematics, University of Colorado, USA, 2003.

27. Sasaki, D., Obayashi, S., and Nakahashi, K., "Navier-Stokes Optimization of Supersonic Wings with Four Objectives Using Evolutionary Algorithm," Journal of Aircraft, Vol. 39, (4), July-August 2002, pp. 621-629.

28. Macmillan, W. L., Development of a Module Type Computer Program for the Calculation of Gas Turbine Off Design Performance, Ph.D. thesis, Department of Power and Propulsion, Cranfield University, 1974.

29. Kulfan, B. M., "Recent Extensions and Applications of the 'CST' Universal Parametric Geometry Representation Method," Aeronautical Journal, Vol. 114, (1153), March 2010, pp. 157-176. 
30. Zhu, F. and Qin, N., "Intuitive Class/Shape Function Parameterization for Airfoils," AIAA Journal, Vol. 52, (1), January 2014, pp. 17-25.

31. Ansys Inc., 275 Technology Drive, Canonsburg, PA 15317, ANSYS ICEM CFD Tutorial Manual.

32. Ansys Inc., 275 Technology Drive, Canonsburg, PA 15317, ANSYS FLUENT User's Guide.

33. Lorenzen, T. and Anderson, V., Design of Experiments, a no-name approach, Marcel Dekker Inc., New York, NY, 1993.

34. Bayraktar, H. and Turalioglu, F., "A Kriging-based approach for locating a sampling site in the assessment of air quality," Stochastic Environmental Research and Risk Assessment, Vol. 19, 2005, pp. 301-305.

35. Deb, K., Pratap, A., Agarwal, S., and Meyarivan, T., "A Fast and Elitist Multiobjective Genetic Algorithm: NSGA-II," EEE Transactions on Evolutionary Computation, Vol. 6, (2), April 2002, pp. 182 - 197.

36. Fan, J. and Gijbels, I., Local Polynomial Modelling and Its Applications: Monographs on Statistics and Applied Probability 66, CRC Press, Boca Raton, FL 33487, USA, March 1996.

37. Bremner, F. J., Gotts, S. J., and Denham, D. L., "Hinton diagrams: Viewing connection strengths in neural networks," Behavior Research Methods, Instruments, $\mathcal{E}$ Computers, Vol. 26, (2), June 1994, pp. 215218.

38. Goulos, I., Stankowski, T., Otter, J., MacManus, D., Grech, N., and Sheaf, C., "Aerodynamic Design of Separate-Jet Exhausts for Future Civil Aero-Engine, Part 1: Parametric Geometry Definition and CFD Approach," ASME J. Eng. Gas Turbines and Power, Vol. 138, (8), August 2016, pp. 081201.

39. Goulos, I., Otter, J., Stankowski, T., MacManus, D., Grech, N., and Sheaf, C., "Aerodynamic Design of Separate-Jet Exhausts for Future Civil Aero-Engines, Part 2: Surrogate Modeling and Optimization,” ASME J. Eng. Gas Turbines and Power, Vol. 138, (8), August 2016, pp. 081202 .

40. Kulfan, B. M., "Universal Parametric Geometry Representation Method," Journal of Aircraft, Vol. 45, (1), January-February 2008, pp. 142-158.

41. Pachidis, V., Pilidis, P., Marinai, L., and Templalexis, I., "Towards a full two dimensional gas turbine performance simulator," Aeronautical Journal, Vol. 111, (1121), 2007, pp. 433-442.

42. Lee, Y.-S., Ma, Y., and Jegadesh, G., "Rolling-Ball Method and Contour Marching Approach to Identifying Critical Regions for Complex Surface Machining," Computers in Industry, Vol. 41, (2), March 2000, pp. 163-180.

43. Olsson, A., Sandberg, G., and Dahlblom, O., "On Latin hypercube sampling for structural reliability analysis," Structural Safety, Vol. 25, No. 1, 2003, pp. $47-68$.

44. Kohavi, R., "A Study of Cross-Validation and Bootstrap for Accuracy Estimation and Model Selection," Proceedings of the Fourteenth International Joint Conference on Artificial Intelligence, Vol. 2, 1995, p. 11371143.

45. Gunston, B., Jane's Aero-engines, Jane's Information Group, 1996.

46. Hotelling, H., "New Light in the Correlation Coefficient and its Transforms," Journal of the Royal Statistical Society, Vol. 15, (2), No. 193-232, 1953.

47. Bonett, D. G. and Wright, T. A., "Sample Size Requirements for Estimating Pearson, Kendall and Spearman Correlations," Psychometrika, Vol. 65, (1), January 2000, pp. 2328. 
2017-09-08

\section{Design optimisation of separate-jet} exhausts for the next generation of civil aero-engin

Goulos, loannis

ISABE

Goulos I, Otter J, Stankowski T, et al., (2017) Design optimisation of separate-jet exhausts for the next generation of civil aero-engines. Proceedings of 23rd International Symposium for Air-Breathing Engines - ISABE 2017, 4-8 September 2017, Manchester, UK https://dspace.lib.cranfield.ac.uk/handle/1826/12564

Downloaded from Cranfield Library Services E-Repository 